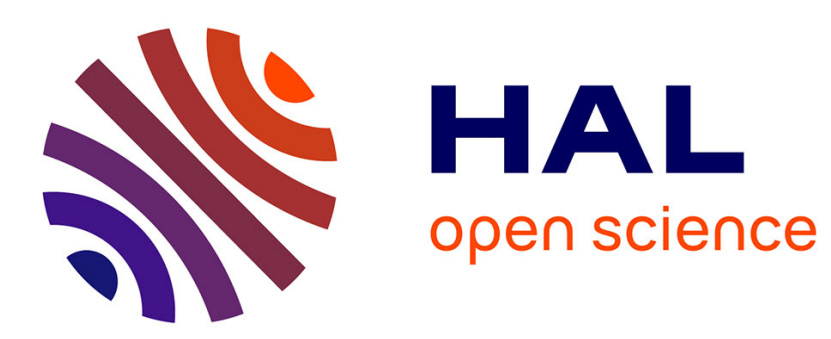

\title{
EXAFS investigations of iodine-doped carbon nanotubes
}

Thierry Michel, Laurent Alvarez, Jean-Louis Sauvajol, Robert Almairac, Raymond Aznar, Jean-Louis Bantignies, O. Mathon

\section{To cite this version:}

Thierry Michel, Laurent Alvarez, Jean-Louis Sauvajol, Robert Almairac, Raymond Aznar, et al.. EXAFS investigations of iodine-doped carbon nanotubes. Physical Review B: Condensed Matter and Materials Physics (1998-2015), 2006, 73 (19), pp.195419. 10.1103/PhysRevB.73.195419 . hal00498071

\section{HAL Id: hal-00498071 \\ https://hal.science/hal-00498071}

Submitted on 13 Dec 2019

HAL is a multi-disciplinary open access archive for the deposit and dissemination of scientific research documents, whether they are published or not. The documents may come from teaching and research institutions in France or abroad, or from public or private research centers.
L'archive ouverte pluridisciplinaire HAL, est destinée au dépôt et à la diffusion de documents scientifiques de niveau recherche, publiés ou non, émanant des établissements d'enseignement et de recherche français ou étrangers, des laboratoires publics ou privés. 


\title{
EXAFS investigations of iodine-doped carbon nanotubes
}

\author{
T. Michel, L. Alvarez, J-L. Sauvajol, R. Almairac, R. Aznar, and J-L. Bantignies \\ Laboratoire des Colloides, Verres et Nanomatériaux (UMR CNRS 5587), Université Montpellier II, \\ 34095 Montpellier Cedex 5, France \\ O. Mathon \\ ESRF, Rue Jules Horowitz, BP 220, 38043, Grenoble Cedex, France
}

(Received 20 February 2006; published 23 May 2006)

\begin{abstract}
We report an $x$-ray absorption fine structure study at the iodine- $K$ edge of the local structure in iodine-doped carbon nanotubes. The iodine-carbon host interaction is shown to be weaker in multiwalled carbon nanotubes (MWNTs) than in single-walled carbon nanotubes (SWNTs). Iodine species are only localized at the surface of the external tube for MWNTs, whereas iodine species enter inside SWNTs. For doped SWNTs, both the experimental and the theoretical EXAFS spectra allow us to establish the structure of the iodine chain as disordered pentaiodide at the saturation level.
\end{abstract}

DOI: 10.1103/PhysRevB.73.195419

PACS number(s): 78.30.Na, 78.70.En

\section{INTRODUCTION}

The tuning of the carbon nanotube electronic properties has been found to be possible by creating charge transfer compounds analogous to graphite intercalation compounds. ${ }^{1}$ Graphite and single-walled carbon nanotubes (SWNTs) have been shown to be amphoteric as they can be doped with either donors $(\mathrm{K}, \mathrm{Rb})$ or acceptors $\left(\mathrm{Br}_{2}\right){ }^{2}$

Iodine has been successfully intercalated into SWNTs. ${ }^{3}$ However, the intercalation site and the form of the doping species are still a controversial point. Raman results suggest charged polyiodide chains $\mathrm{I}_{5}{ }^{-}$and $\mathrm{I}_{3}{ }^{-}$to be distributed throughout the iodine-doped SWNTs (I-SWNTs). ${ }^{3}$ Based on a comparison between the pressure behavior of the Raman modes of I-SWNTs, it has been suggested that $\left(\mathrm{I}_{n}\right)^{-}$molecules might reside both in the interstitial channels and inside the tubes in SWNTs bundles. ${ }^{5}$ In the same way, resolution $Z$-contrast scanning transmission electron microscopy images have revealed the incorporation of iodine atoms in the form of helical chains inside the SWNTs. ${ }^{6}$ Nevertheless, the overall composition $\mathrm{IC}_{12}$ suggests that substantial iodine species are also incorporated in the interstitial position between tubes in the bundles ${ }^{6}$ as many tubes are unfilled. Furthermore, $\mathrm{x}$-ray and neutron diffraction investigations of I-SWNTs showed that $\left(\mathrm{I}_{n}\right)^{-}$species are mainly localized inside the tubes. ${ }^{7}$ Nevertheless, the detailed knowledge about the structure of iodine-doped carbon nanotubes, especially the local geometry around the iodine ions, the number of nearest neighbors of iodine ions, and the distance between iodine ions and carbon atoms are still open questions.

Concerning the iodine intercalation of multiwalled nanotubes (MWNTs), the doping process is probably the most unclear. A Raman study reveals the presence of iodine species $\left(\mathrm{I}_{n}\right)^{-}$in iodine-doped MWNTs (I-MWNTs) but cannot give a direct evidence of a charge transfer since the tangential modes of the nanotubes seem to be not affected by the presence of iodine chains. ${ }^{4}$ Considering the stoichiometry of such samples $\left(\mathrm{IC}_{200}\right)$, iodine is assumed to be located only inside the tubes of different inner diameters. ${ }^{4}$ This assumption is consistent with the fact that the spacing between graphene sheets in MWNTs is almost the same as in graphite which cannot accommodate iodine species. ${ }^{1}$ It appears that both the intercalation site and the structure of the doping species in carbon nanotubes are still an open question.

In the aim to light these points, the x-ray absorption spectroscopy (XAS) appears to be a very promising technique to measure the molecular parameters of charged polyiodide chains in carbon nanotubes, especially the local geometry around the iodine ions, the number of the nearest neighbors of iodine ions, and the distance between iodine ions and carbon atoms. XAS has been successfully used to study the local structure around alkali ions inserted into SWNTs bundles. ${ }^{8}$ Atomic selectivity and short range sensitivity allow us to measure the I-I EXAFS response in different phases. The I $K$ edge is the most appropriate absorption threshold to be studied due to the high energy $\left(E_{0}=33.169 \mathrm{keV}\right)$, leading to a high penetration depth. Experimental work on polyiodine anions, iodine-doped pol(octylthiophene), and $\mathrm{I}_{2}$ in different states of matter were carried out using the extended $\mathrm{x}$-ray absorption fine structure (EXAFS) technique to study the form of the iodine species. ${ }^{9,10}$ In the present paper, we report the results of an EXAFS investigation of the $\left(\mathrm{I}_{n}\right)^{-}$ structure in single-walled and multiwalled carbon nanotubes. The different iodine-doped carbon nanostructures (I-MWNTs and I-SWNTs) from different origins are doped using exactly the same procedure. The behavior observed for multiwalled carbon nanotubes turns out to be radically different from the one observed for I-SWNTs. We present herein direct proofs of selective doping depending on the structural properties of carbon nanostructures.

\section{EXPERIMENT}

\section{A. Samples}

Three different kinds of SWNTs samples were used. SWNTs were prepared in our laboratory by the electric arc method. ${ }^{11}$ Commercial HIPCO $^{12}$ and carbon solution samples ${ }^{13}$ were also studied. In all cases, the raw materials were characterized by $\mathrm{x}$-ray diffraction in order to select only 
parts of the samples with an intense (10) reflection peak of the bundles. Consequently, all the selected samples contain a large amount of bundles. MWNTs were also synthesized by the electric arc method. ${ }^{14}$ After outgassing and annealing the samples at $250{ }^{\circ} \mathrm{C}$ for $24 \mathrm{~h}$ under a dynamical vacuum, iodine doping of the carbon materials is achieved by immersing the nanomaterials in molten iodine in an evacuated quartz tube at a temperature $T=140{ }^{\circ} \mathrm{C}$ for several days to perform a saturation doping. ${ }^{3}$ Most of the iodine in excess is removed by the cold point method. However, x-ray diffractions performed after the sample preparation show that crystalline $\mathrm{I}_{2}$ particles are present in the powder. Consequently, after doping, materials were separated into two parts: a reference set of materials and a second set washed by ethanol several times in order to remove all the iodine in excess.

\section{B. Experimental setup}

Low temperature $(10 \mathrm{~K})$ XAS experiments are performed at the beamline BM 29 at the ESRF facility. Spectra were measured at the iodine $K$ edge $\left(E_{0}=33.169 \mathrm{keV}\right)$ in the transmission mode with a double $\mathrm{Si}(311)$ crystal monochromator. Ionization chambers were used to detect the signals.

\section{Data analysis}

The EXAFS oscillations $\chi(k)$ are extracted for XAS data using a standard procedure. ${ }^{15,16}$ After the background subtraction and edge step normalization of the absorption data, the $\chi(k)$ signals are obtained. The radial structure function $\mathrm{FT}\left(k^{3} \chi(k)\right)$ is obtained by the Fourier transform of $k^{3} \chi(k)$. The Fourier transform window has been chosen to start at a very low $k\left(\simeq 1.7 \AA^{-1}\right)$ since the scattering amplitude of carbon atoms is rather high in the $1.5-5 \AA^{-1} k$ range. According to $a b$ initio calculations using the FEFF program, ${ }^{19}$ no multiple scattering is predicted in such samples.

\section{RESULTS}

Figure 1 shows the EXAFS oscillations $k \chi(k)$ of iodinedoped carbon nanostructures, respectively, I-MWNTs and I-SWNTs. They all display a good signal up to $17 \AA^{-1}$. The EXAFS spectra of I-MWNTs and I-SWNTs display differences around 3.2, 5.6, 7, and 9.2 $\AA^{-1}$ (arrows in Fig. 1). The local arrangement of iodine in I-MWNTs is well simulated using ab initio FEFF calculations by considering the structure of the $\mathrm{I}_{2}$ crystal up to $5 \AA$ around iodine (Fig. 2). This result appears surprising since ionic $\left(\mathrm{I}_{n}\right)^{-}$species are evidenced by micro-Raman spectroscopy in the low frequency range of all nanotubes. ${ }^{3,4,7}$ This discrepancy could be due to excessive iodine present in our macroscopic samples as neutral $\mathrm{I}_{2}$ crystallites. Indeed, $\mathrm{x}$-ray absorption spectroscopy probes the bulk of the sample whereas micro-Raman probes a small depth of well-dispersed clusters. Because $\mathrm{I}_{2}$ crystallites evaporate very quickly in contact with air, the Raman response is only given by $\left(\mathrm{I}_{n}\right)^{-}$species interacting with the tubes.

Unlike I-MWNTs, the $\mathrm{I}_{2}$ crystal structure does not allow us to simulate properly the local arrangement of iodine in

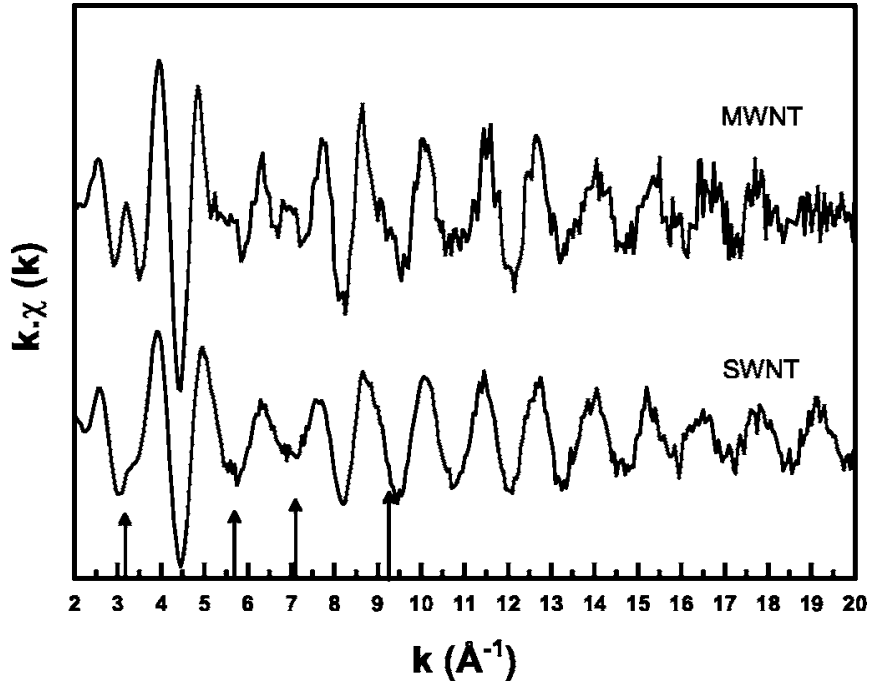

FIG. 1. EXAFS spectra at the iodine $K$ edge of the unwashed (a) I-MWNTs and (b) I-SWNTs.

I-SWNTs. The slight differences between EXAFS oscillations of I-MWNTs and I-SWNTs are probably due to a peculiar intercalation site available in I-SWNTs but not in I-MWNTs. The difference between I-MWNTs and I-SWNTs can be explained either in terms of distinct intercalation sites between both samples or in terms of different interaction energies between iodine and carbon nanostructures. Concerning the interaction energies, thermogravimetric analysis experiments have shown that different energies are required to remove iodine from I-MWNTs and I-SWNTs, respectively. Indeed, the loss of iodine begins at room temperature for MWNTs ${ }^{4}$ and only at $100{ }^{\circ} \mathrm{C}$ for SWNTs. ${ }^{3}$ In order to get rid of excess iodine $\mathrm{I}_{2}$ crystal clusters which are not intercalated, all the samples were washed with ethanol. We found out that iodine was no longer present in I-MWNTs as evidenced by XAS since no absorption edge is observed after washing. Since iodine can be removed easily by washing, the interactions between iodine and the carbon network are supposed to be weak in I-MWNTs. By contrast, after washing,

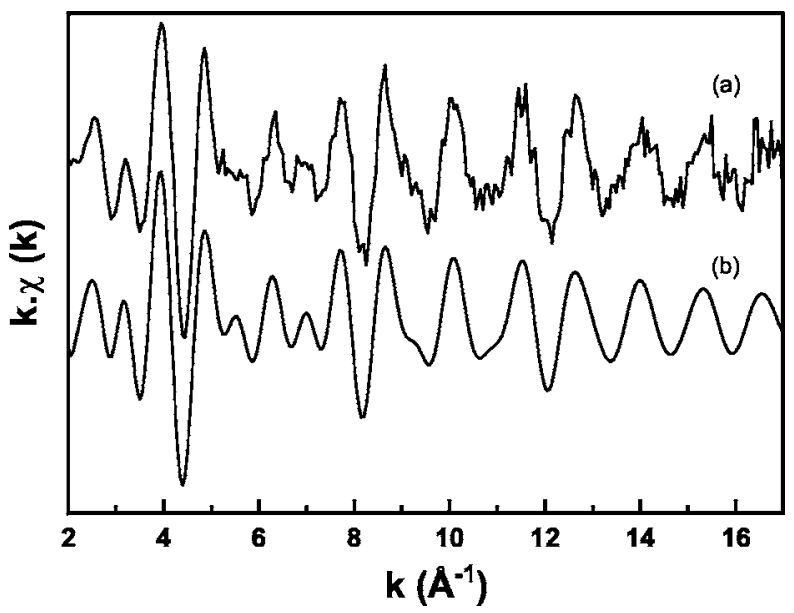

FIG. 2. Experimental EXAFS signal of the I-MWNTs sample (a) and theoretical $a b$ initio spectrum of $\mathrm{I}_{2}$ crystal clusters (b). 


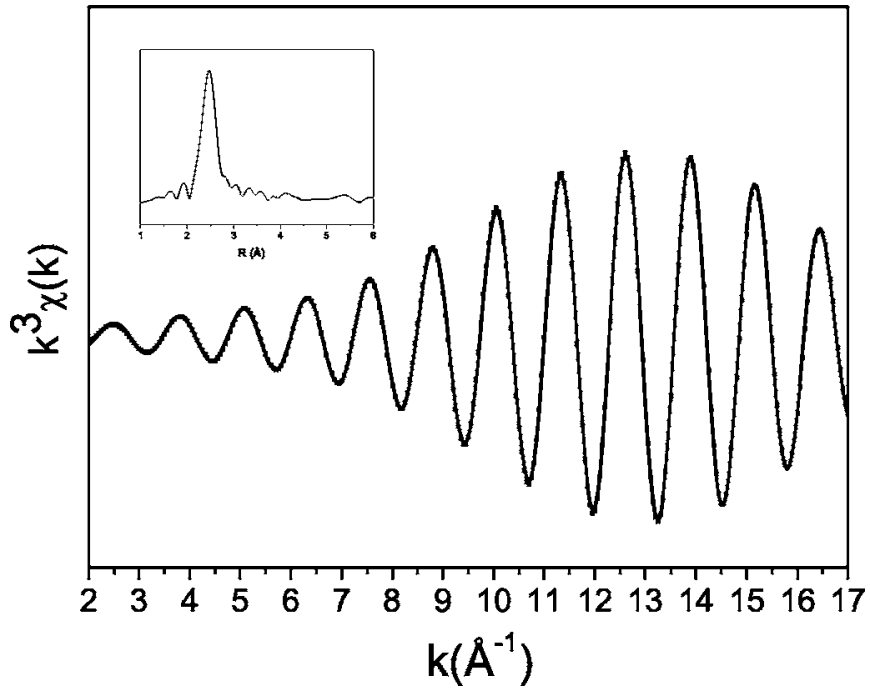

FIG. 3. Back Fourier transform of the experimental spectrum and the fit taking into account one shell of iodine and one shell of carbon; inset: Fourier transform $\mathrm{FT}\left[\mathrm{k}^{3} \chi(k)\right]$ of the experimental EXAFS spectrum; note that the difference between the peak position $(2.5 \AA)$ and the real I-I distance $(2.73 \AA)$ is due to a phase term.

an iodine absorption edge is still observable on washed I-SWNTs, meaning that iodine is still present in this type of carbon nanostructures. Thus, we could consider that specific sites in I-SWNTs prevent iodine removing. The interstitial site between three SWNTs within the bundles could explain this behavior. However, according to the large van der Waals iodine diameter $(\simeq 4 \AA$ ), a penetration of iodine chains in the interstitial site has a low probability [available space $\simeq 2.6 \AA$ in diameter for $(10,10)$ SWNTs, taking into account the Van der Waals radius]. The other sites available (outside and inside the tube for MWNTs, outside the bundles and inside the tube for SWNTs) are present in both samples, with a number of internal sites being much more important in SWNTs. It was already shown for MWNTs (even with a few walls) that only the outer tube is affected by the intercalation process. ${ }^{17}$ As $\mathrm{I}_{2}$ in the molten phase is known to dissociate into very oxidizing iodium $\mathrm{I}^{+}$species and polyiodide $\mathrm{I}_{n}{ }^{-6}$, it is assumed that $\mathrm{I}^{+}$attacks the tube ends and the defect sites, making possible the entrance of polyiodide species inside tubes. Hence, the SWNTs, because of their unique layer, are probably much easier to open with respect to MWNTs for which several layers have to be destroyed. Thus, since iodine is completely removed under washing in MWNTs, we suggest that iodine is only adsorbed at the surface of the outer shell in this kind of nanostructures, in good agreement with x-ray diffraction ${ }^{7}$ and Raman spectroscopy results. ${ }^{17}$ Hence, we assume that iodine can penetrate SWNTs but not MWNTs.

Since the EXAFS signal of I-doped nanotubes is dominated by $\mathrm{I}_{2}$ crystal clusters, even for I-SWNTs, we will consider in the following, only washed SWNTs samples. Concerning the local arrangement of iodine in washed I-SWNTs, the Fourier transform $\operatorname{FT}\left(k^{3} \chi(k)\right)$ of the experimental EXAFS spectrum as a function of a nonphase corrected radial distance is shown in the inset of Fig. 3. The $\mathrm{FT}\left(k^{3} \chi(k)\right)$ gives
TABLE I. Structural parameters deduced from the least squares fit of the first shells of the I-doped SWNTs samples. $N$ is the coordination number, $R$ the fitted I-I or I-C distance, $\sigma$ the DebyeWaller factor and $\Delta E$ the energy shift.

\begin{tabular}{lcc}
\hline \hline & Iodine shell & Carbon shell \\
\hline$N$ & 0.8 & 2.2 \\
$\sigma^{2}$ & 0.0016 & 0.02 \\
$\Delta E$ & 12.11 & 2.8 \\
$R$ & 2.74 & 3.02 \\
\hline \hline
\end{tabular}

rise to only one peak located around $2.74 \AA$. The structural parameters of the first coordination shell of I-SWNTs are fitted using the theoretical electronic parameters (backscattered amplitudes, phases, and the mean free path) calculated by FEFF. By considering only iodine first neighbors, the agreement between the filtered experimental EXAFS signal and the fit is not really satisfactory. There are some discrepancies at low $k$. As the backscattered amplitude contribution of the carbon atoms is important at low $k$, a second set of fits is performed taking into account both the iodine neighbors and the carbon neighbors. In this case, a very good agreement between the experimental data and the fit is obtained as shown in Fig. 3. The structural parameters extracted, respectively, the number of neighbors $N$, the interatomic distance $R$, the square of the Debye-Waller factor $\sigma^{2}$, are given in Table I. We can first note that the Debye-Waller factor associated with the disorder in the structure is quite low in the case of the iodine-iodine distance and quite high for the iodine-carbon distance. Depending on the localization of the iodine chains with respect to the carbon network, we expect a distribution of iodine-carbon distances and then a large Debye Waller factor $\sigma^{2}\left(0.02 \AA^{2}\right)$. EXAFS experiments on an iodine-doped polymer determined the I-I distance and the number of neighbors as a function of the chain length. ${ }^{10}$ The $\left(\mathrm{I}_{3}\right)^{-}$chain is formed with an I-I first distance of $2.9 \AA$ whereas the number of neighbors is 1.2 and the $\mathrm{I}_{5}{ }^{-}$chain leads to a first I-I distance of $2.73 \AA$ and a coordination number close to 0.85 . In our I-SWNTs samples, we found out a number of neighbours of about 0.8 and an I-I distance around $2.74 \AA$. These structural parameters suggest the presence of $\mathrm{I}_{5}{ }^{-}$chains in our sample doped at saturation. This result is in good agreement with the work of Aasmundtveit et $a l .{ }^{10}$ and Gregorian et al..${ }^{4}$ since they assume the presence of only $\mathrm{I}_{5}{ }^{-}$chains at a saturation level for polymer and SWNTs, respectively. The structural model for this $\mathrm{I}_{5}{ }^{-}$chain suggests two $\mathrm{I}_{2}$ molecules surrounding an $\mathrm{I}^{-}$ion. Nevertheless, the long bond in pentaiodine (between the central $\mathrm{I}^{-}$and the external $\mathrm{I}_{2}$ molecules) should give rise to a signal around $3.2-3.3 \AA$. This signal is not observed in our samples, probably because of large fluctuations in this bond (due to the presence of the extra electron). Other authors came to the same conclusion for iodine-doped polymers. ${ }^{10}$

However, unlike those EXAFS results, Raman spectroscopy experiments also suggest the presence of $\mathrm{I}_{3}{ }^{-}$chains. Indeed, the couple of peaks in the low frequency region, around 110 and $172 \mathrm{~cm}^{-1}$ are generally assigned to the response of $\mathrm{I}_{3}{ }^{-}$and $\mathrm{I}_{5}{ }^{-}$chains, respectively. ${ }^{3-5,7}$ However, this 


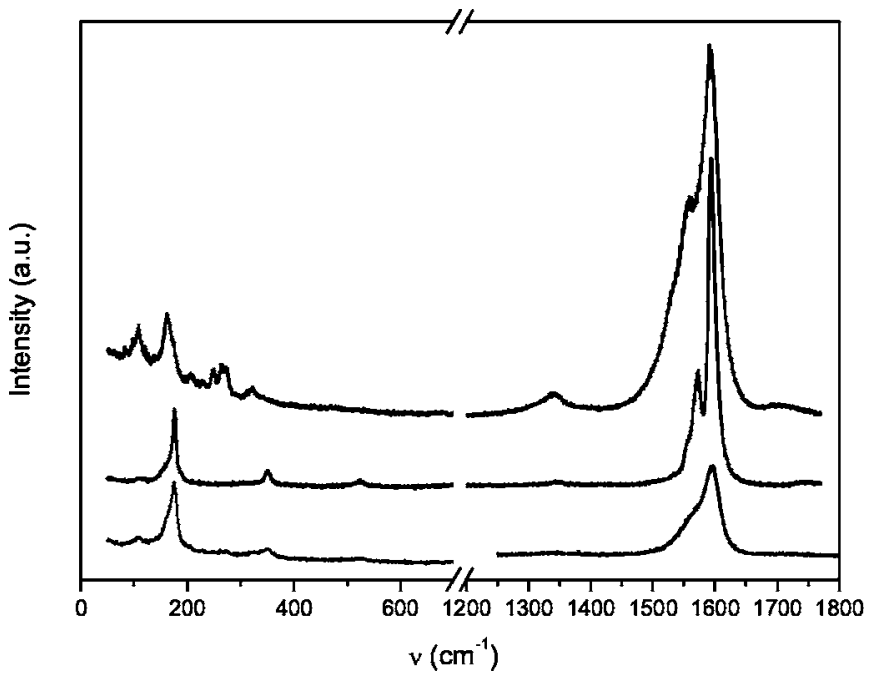

FIG. 4. Raman spectra of iodine doped SWNTs: Top, carbon solution samples; bottom and middle, HIPCO samples. MicroRaman spectra were measured using the $514.5 \mathrm{~nm}$ excitation line.

assumption relies only on the work of Teitelbaum and co-workers ${ }^{18}$ in which the Raman signal is ascribed to $\mathrm{I}_{2}$ molecules weakly interacting with distorted $\mathrm{I}_{3}{ }^{-}$chains. But depending on the type of molecules (respectively, one and two $\mathrm{I}_{3}{ }^{-}$chains interacting with only one $\mathrm{I}_{2}$ molecule), the frequencies associated with iodine modes might shift of about $10 \mathrm{~cm}^{-1}$ (respectively, 104 and $162 \mathrm{~cm}^{-1}$ or 110 and $\left.172 \mathrm{~cm}^{-1}\right) .{ }^{18}$ Raman spectra of our I-SWNTs are shown in Fig. 4. The low frequency region is sample dependent. The top spectrum displays a couple of peaks around 108 and $162 \mathrm{~cm}^{-1}$, whereas the other two have peaks around 110 and $175 \mathrm{~cm}^{-1}$. In addition, the Raman spectra depend on the excitation wavelength, so that it looks quite tricky to assign unambiguously these peaks to specific $\mathrm{I}_{n}{ }^{-}$chains. Consequently, we assume that Raman spectroscopy can give direct proofs on the charge transfer but only some insights about the structure of the $\mathrm{I}_{n}{ }^{-}$chains intercalated.

In order to confirm our assumptions about the presence of $\mathrm{I}_{5}{ }^{-}$chains exclusively, we also performed $a b$ initio FEFF simulations to better discuss both the iodine chain structure and its localization within the bundles. EXAFS spectra simulations are shown in Fig. 5. The $\mathrm{I}_{3}{ }^{-}$chain gives rise to an EXAFS response in total disagreement with the experimental data. Consequently, as previously assumed from the fit of our data, we do not observe $\mathrm{I}_{3}{ }^{-}$chains in our samples. The symmetric $\mathrm{I}_{5}{ }^{-}$chain with its two I-I distances (centered at $2.73 \AA$ and around $3.25 \AA$ ) also gives rise to a signal in complete disagreement with the experimental data (Fig. 5), so that the presence of the ordered symmetric $\mathrm{I}_{5}{ }^{-}$chain in our samples is ruled out. However, if we consider an $\mathrm{I}_{5}^{-}$chain (noted $\mathrm{I}_{5} R$ in Fig. 5) with a large distribution of I-I second neighbors (due to the presence of the delocalized extra charge), the large fluctuations kill out the response of the second I-I distance. Under this assumption, the experimental EXAFS response is quite well simulated (in terms of the phase and amplitude). The signal is actually perfectly reproduced in the 5.5-17 $\AA^{-1} k$ range, where the response is dominated by the I-I distance. Nevertheless, we can notice some slight dis-

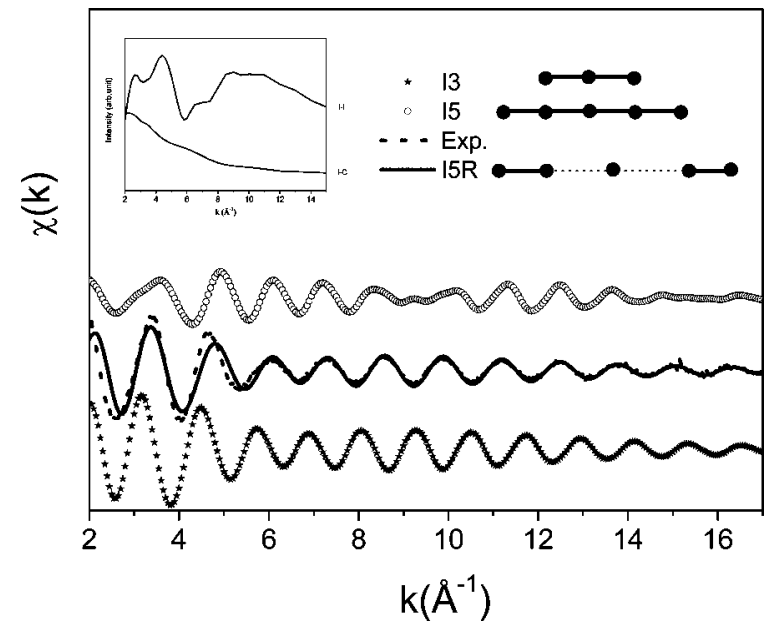

FIG. 5. Experimental and simulated EXAFS spectra of several probable environments around the iodine absorbing atom. Inset: back scattering amplitudes of the iodine-iodine and iodine-carbon pair of atoms; drawings of the different $\left(\mathrm{I}_{n}\right)^{-}$chains.

crepancies between both EXAFS spectra in the low $k$ region $\left(k<5 \AA^{-1}\right)$. These differences probably arise from the carbon atom environment around the iodine species which is not taken into account. Indeed, the carbon atoms contribution is mainly expected in the $1-5 \AA^{-1} k$ range where the backscattering amplitude reaches a maximum (inset Fig. 5). Consequently, we assume that the experimental EXAFS signal essentially arises from the very disordered $\mathrm{I}_{5}{ }^{-}$chains inside SWNTs.

The question now concerns the different possible insertion sites of the iodine chains in the nanotube host matrix. The interstitial site, which is usually reported as one of the preferential sites of the iodine species insertion in I-SWNTs bundles, seems to be ruled out from the quantitative analysis of the EXAFS results. If iodine was localized, respectively, in between two or three tubes within the bundle, the number of carbon neighbors would be, respectively, 12 or 18 for a $(10,10)$ nanotube bundle in the ideal case. In a normal case, this number would be lower but significantly higher than 2 . Thus, the first argument to rule out this site is that the number of carbon neighbors should be larger than the one found (around 2, see Table I). In addition, an I-C distance of about $3 \AA$ is incompatible with a position between tubes where the distance available is not large enough. In addition, the extremely weak expansion of the 2D triangular lattice under the intercalation of iodine in SWNTs bundles, stated from the neutrons diffraction experiment ${ }^{7}$ rules out an intercalation site within the interstitial sites of bundles. On the other hand, ethanol washing removes the iodine species at the surface of MWNTs. This result implies that the surface of SWNTs bundles is also free of iodine species after washing. Consequently, we conclude that the iodine $\mathrm{I}_{5}{ }^{-}$chains are mainly located inside SWNTs.

\section{CONCLUSION}

To summarize, we have shown that EXAFS investigations on iodine-doped carbon nanostructures can bring very useful 
information on the structural properties of these compounds. We have first compared the iodine intercalation process in different carbon nanostructures. The study has evidenced two different kinds of interactions between the iodine chains and the carbon network, a weak interaction in I-MWNTs and a stronger one in I-SWNTs. The EXAFS results state the pres- ence of $\left(\mathrm{I}_{n}\right)^{-}$species inside the tubes for I-SWNTs. The latter one is still occupied after washing, meaning that SWNTs protect the iodine species. We also find out that iodine is mainly present as disordered $\mathrm{I}_{5}{ }^{-}$chains in the I-SWNTs samples, in good agreement with other works related to samples doped at the saturation level.
${ }^{1}$ M. S. Dresselhaus and G. Dresselhaus, Adv. Phys. 30, 139 (1981).

${ }^{2}$ R. S. Lee, H. J. Kim, J. E. Fischer, A. Thess, and R. E. Smalley, Nature (London) 388, 257 (1997).

${ }^{3}$ L. Grigorian, K. A. Williams, S. Fang, G. U. Sumanasekera, A. L. Loper, E. C. Dickey, S. J. Pennycook, and P. C. Eklund, Phys. Rev. Lett. 80, 5560 (1998).

${ }^{4}$ W. Zhou, S. Xie, L. Sun, D. Tang, Y. Li, Z. Liu, L. Ci, X. Zou, G. Wang, P. Tan, X. Dong, B. Xu, and B. Zhao, Appl. Phys. Lett. 80, 2553 (2002).

${ }^{5}$ U. D. Venkateswaran, E. A. Brandsen, M. E. Katakowski, A. Harutyunyan, G. Chen, A. L. Loper, and P. C. Eklund, Phys. Rev. B 65, 054102 (2002).

${ }^{6}$ X. Fan, E. C. Dickey, P. Eklund, K. Williams, L. Grigorian, R. Buczko, S. T. Pantelides, and S. J. Pennyccok, Phys. Rev. Lett. 84, 4621 (2002).

${ }^{7}$ N. Bendiab, R. Almairac, S. Rols, R. Aznar, J. L. Sauvajol, and I. Mirebeau, Phys. Rev. B 69, 195415 (2004).

${ }^{8}$ J. L. Bantignies, L. Alvarez, R. Aznar, R. Almairac, J. L. Sauvajol, L. Duclaux, and F. Villain, Phys. Rev. B 71, 195419 (2005).

${ }^{9}$ H. Sakane, T. Mitsui, H. Tanida, and I. Watanabe, J. Synchrotron Radiat. 8, 674 (2001).
${ }^{10}$ K. E. Aasmundtveit, E. J. Samuelsen, C. Steinsland, C. Meneghini, and A. Filipponi, Synth. Met. 101, 363 (1999).

${ }^{11}$ C. Journet, W. Maser, P. Bernier, A. Loiseau, M. L. de la Chapelle, S. Lefrant, P. Deniard, R. Lee, and J. E. Fischer, Nature (London) 388, 756 (1997).

${ }^{12}$ P. Nikolaev, M. Bronikowski, R. Bradley, F. Rohmund, D. Colbert, K. Smith, and R. Smalley, Chem. Phys. Lett. 313, 91 (1999).

${ }^{13} \mathrm{http}: / /$ carbonsolution.com(P2-SWNT samples)

${ }^{14}$ P. Piedigrosso, Z. Konya, J. F Colomer, A. Fonseca, G. Van Tendeloo, and J. B. Nagy, Phys. Chem. Chem. Phys. 1, 163 (2000).

${ }^{15} \mathrm{D}$. Koningsberger and R. Prins, X-ray Absorption Principles Applications, Techniques of EXAFS, SEXAFS and XANES (Wiley, New York, 1988).

${ }^{16}$ Boon K. Teo, EXAFS: Basic Principles and Data Analysis (Springer-Verlag, Berlin, 1985).

${ }^{17}$ J. Cambedouzou, J. L. Sauvajol, A. Rahmani, E. Flahaut, A. Peigney, and C. Laurent, Phys. Rev. B 69, 235422 (2004).

${ }^{18}$ R. C. Teitelbaum, S. L. Ruby, and T. J. Marks, J. Am. Chem. Soc. 101, 25 (1979).

${ }^{19}$ A. L. Ankudinov, B. Ravel, J. J. Rehr, and S. D. Conradson, Phys. Rev. B 58, 7565 (1998). 